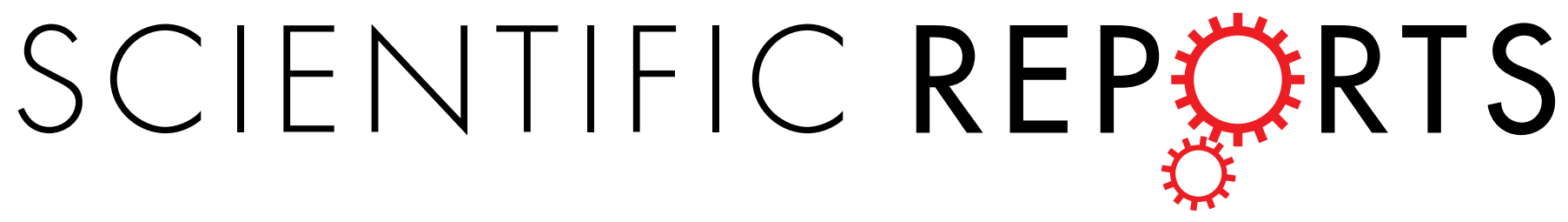

\title{
Erratum: A Novel Audiovisual Brain-Computer Interface and Its Application in Awareness Detection
}

Fei Wang, Yanbin He, Jiahui Pan, Qiuyou Xie, Ronghao Yu, Rui Zhang \& Yuanqing Li

Scientific Reports 5:9962; doi: 10.1038/srep09962; published online 30 June 2015; updated 24 August 2015

This Article contains a typographical error in Affiliation 2. The correct affiliation is listed below:

Coma Research Group, Centre for Hyperbaric Oxygen and Neurorehabilitation, Guangzhou General Hospital of Guangzhou Military Command, Guangzhou 510010, China. 